\title{
Research on Micro-piezoresistive Accelerometer with Slotted Beams
}

\author{
Peng Wang, ${ }^{1,2^{*}}$ Nan Wang, ${ }^{1,2}$ Changming Zhang, ${ }^{1,2}$ Fan Yang, ${ }^{1,2}$ and Ruijie Zhao ${ }^{1}$ \\ ${ }^{1}$ Mechanical Engineering Institute, Shaanxi University of Technology, \\ Chaoyang Road, Han Zhong, Shaanxi 723000, China \\ ${ }^{2}$ Shaanxi Key Laboratory of Industrial Automation, Shaanxi University of Technology, \\ Chaoyang Road, Han Zhong, Shaanxi 723000, China
}

(Received September 1, 2020; accepted November 9, 2020)

Keywords: accelerometer, stress concentration, piezoresistive, slotted beam

To meet the requirements of vibration sensors in spindle vibration measurement systems, a new micro-electromechanical systems (MEMS) silicon piezoresistive accelerometer with a slotted eight-beam (SEB) structure was designed and developed. The proposed structure was designed to improve the trade-off between the sensitivity and the natural frequency of a piezoresistive accelerometer, which is made possible by incorporating slots into the eightbeam (EB) structure. To obtain the optimal parameters of the designed sensor, the mechanism by which the slot location and size influence the performance of the designed structure was studied and analyzed by finite element simulation (FEM). The developed sensor and EB structure sensor were fabricated on an n-type single-crystal silicon wafer and packaged for an experiment. The accelerometer prototype was tested in a centrifugal machine and a dynamic calibration system. The experimental results show that the sensitivity of the designed sensor is $2.03 \mathrm{mV} / \mathrm{g}$ and its natural frequency is $14.16 \mathrm{kHz}$. Compared with the structure without slots, the sensitivity was increased by $45 \%$ and the natural frequency remained basically unchanged.

\section{Introduction}

A spindle system is one of the core components of high-speed intelligent machinery, which can achieve high-reliability machining. The vibration of the spindle caused by the dynamic balance and other factors will reduce the accuracy and reliability of machining, so it is necessary to monitor high-frequency signals of the spindle system. ${ }^{(1)}$ As the first component to recognize and convert vibration signals, the vibration picking accuracy of the vibration sensor will directly affect the working accuracy of the whole vibration-measuring system. ${ }^{(2)}$ With the development of intelligent manufacturing equipment and wireless sensor networks, higher requirements are expected for the volume and cost of vibration sensors. However, domestic high-performance sensors are heavily dependent on imports, and it is difficult to meet the demand for the development of intelligent manufacturing equipment. ${ }^{(3-5)}$

With the development of micro-electromechanical systems (MEMS) technology, the use of MEMS technology to process microsensors has become one of the research hotspots *Corresponding author: e-mail: wangpeng1851@163.com https://doi.org/10.18494/SAM.2020.3079 
domestically and abroad. Among them, the MEMS accelerometer has become the main component used for vibration measurement. MEMS accelerators include those based on capacitive, piezoelectric, resonant, piezoresistive, and other conversion principles. The piezoresistive accelerometer, which was the earliest silicon micro-accelerometer to be developed, has been widely used in the field of high-frequency vibration signal measurement owing to its advantages of a simple structure, low power consumption, and a wide operating frequency band. ${ }^{(6)}$

The sensitivity of a piezoresistive accelerometer is mainly determined by the stress at the position of the piezoresistor and piezoresistor coefficient. The former can be realized by optimizing the sensitive structure and the detection circuit. For example, the sensitivity of a sensor can be increased by using the stress concentration effect and by the combination of the large and small beams. ${ }^{(7-9)}$ The latter relies on the development of new materials and new MEMS processes to improve sensor sensitivity by replacing the traditional doped resistance with emerging materials such as polymer composite nanomaterials, graphene, and amorphous carbon films. ${ }^{(10,11)}$ In contrast, because changing the sensitive structure does not depend on the development of new materials, it is more suitable for the development of high-performance accelerometers. $^{(12)}$

The incorporation of stress concentration slots into the sensitive structure was proved effective for enhancing the sensitivity of a sensor. Wang et al. reported a slotted-cantilever accelerometer, which enhanced the sensitivity from less than $1 \mathrm{mV} /(\mathrm{Vg})$ to $5.189 \mathrm{mV} /(\mathrm{Vg}){ }^{(13)}$ In 2012, Zhao et al. successfully developed a piezoresistive accelerometer based on a slotted quad-beam structure, which consisted of a proof mass supported by four thin flexures with slots etched in the middle and had greater sensitivity than the traditional structure. ${ }^{(14)}$ However, the above study did not deeply investigate the mechanism by which the slots affect the sensor performance, and the natural frequency of the sensor decreased after the slots were introduced. How to minimize this effect should be further studied. Therefore, on the basis of the previous research of our research group, a high-performance MEMS piezoresistive accelerometer with a slotted eight-beam (SEB) structure was proposed and developed. The slot size and parameters were optimized by studying the mechanism by which the slot position and size affect the sensor performance. The sensitive structure not only improves the sensitivity of the sensor, but also ensures that the natural frequency of the sensor remains within the usable range. This paper introduces in detail the design of the sensitive structure of the sensor, the optimization of the structure size, the encapsulation of the sensor, and its performance test, and gives the results of a comparison between the sensor and other sensors. The results show that our newly designed sensor has better performance.

\section{Design of Sensitive Structure}

In this paper, an SEB structure based on slot etching in the self-designed eight-beam (EB) structure was proposed and developed. The proposed SEB structure is schematically shown in Fig. 1(a). The piezoresistors are arranged in the sensing beams and connected into a Wheatstone bridge as shown in Fig. 1(b). When acceleration is applied along the $z$ axis (the 


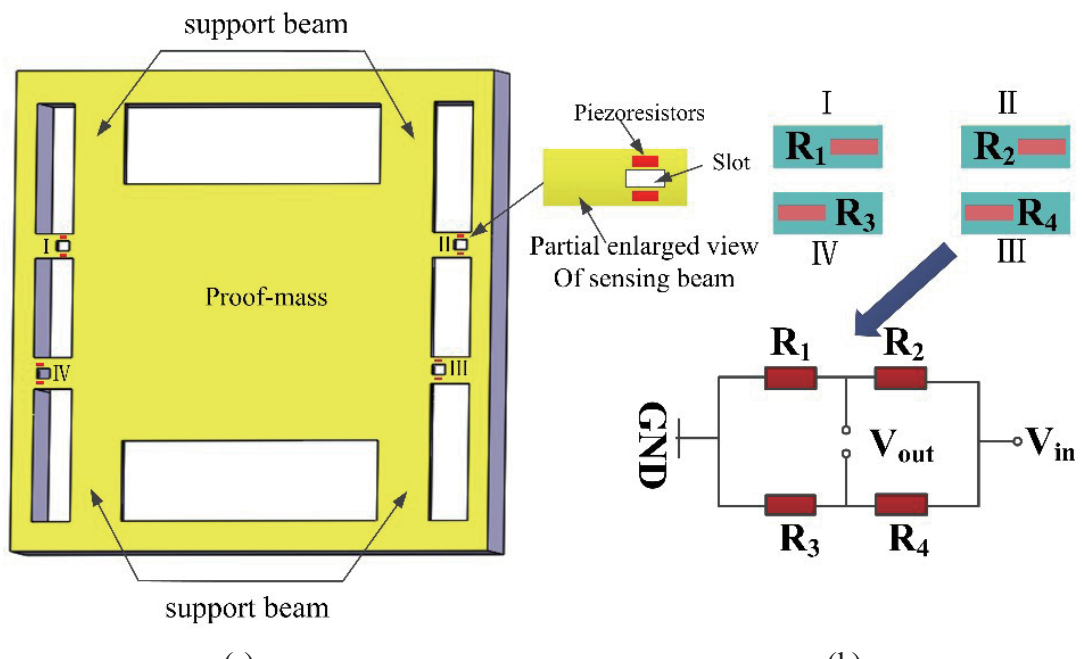

(a)

(b)

Fig. 1. (Color online) Schematic of the accelerometer with the SEB structure: (a) SEB structure; (b) piezoresistors in the sensing beams.

direction normal to the sensor chip), the proof mass will move vertically and the sensing beams will deform. The stress produced by deformation of the sensing beams changes the resistance of the piezoresistors, which leads to the unbalance of the Wheatstone bridge.

Model analysis and static analysis by finite element analysis are used to simulate the variation of the maximum stress and natural frequency with the sizes and positions of the slots. Herein, the Young's modulus is $E=166 \mathrm{GPa}$, the density is $\rho=2331 \mathrm{~kg} / \mathrm{m}^{3}$, the Poisson's ratio is 0.27 , and the applied $\mathrm{G}$ for simulation is $100 \mathrm{~g}$. The sizes of the proof mass, sensing beams, and suspension beams are $3000 \times 2500 \times 380 \mu \mathrm{m}^{3}, 220 \times 100 \times 22 \mu \mathrm{m}^{3}$, and $450 \times 150 \times 22 \mu \mathrm{m}^{3}$, respectively. ${ }^{(15)}$ For convenience, it is assumed that the length of a slot is $M$ times the length of the sensitive beam, its width is $N$ times the width of the sensing beam, its thickness is equal to that of the sensitive beam, and the distance between the slot and the proof mass is $H$ times the length of the sensitive beam. The values of $M, N$, and $H$ are all less than 1 .

To determine the above parameters, the following conditions should be met: (1) The size of the slot must not be larger than the size of the sensitive beam. (2) In accordance with the processing capability of Beijing Microelectronics Research Institute, the minimum piezoresistor width is $8 \mu \mathrm{m}$. The minimum gap between the piezoresistor and the slot is $8 \mu \mathrm{m}$, and the minimum side length of the ohmic contact area is $20 \mu \mathrm{m}$. (3) Since the natural frequency of the sensor will decrease after the introduction of slots, it is necessary to control the attenuation of the natural frequency. To meet the requirements of the project, the designed sensor has a natural frequency higher than $13 \mathrm{kHz}$. Since the width of the slot should consider the positional relationship between the slot, the piezoresistor, and the edge of the sensitive beam at the same time, combining this with the processing capacity of Beijing Microelectronics Research Institute, $N$ was determined to be 0.5 . The effects of $H$ and $M$ on sensor performance are analyzed below. 


\subsection{Effect of $H$ on sensor performance}

Assuming that the length and width of the slot are constant, namely, that $M$ and $N$ are fixed, the static and dynamic characteristics of the sensor are simulated by changing $H$, where $M$ and $N$ are 0.35 and 0.5 , respectively. On the basis of the relationship in Fig. 2 and the MEMS processing capacity, $H$ was varied from 0.05 to 0.6 . Figures 3 and 4 respectively show the influence of $H$ on the sensitivity and natural frequency of the designed sensor. As can be seen from Fig. 3, the maximum stress on the beam is distributed almost symmetrically as a function of $H$, and the closer the slot is to the proof mass or the fixed end, the higher the sensitivity of the sensor. The maximum stress on the sensing beam is $32.4 \mathrm{MPa}$ and the minimum value is 21.1 MPa. This indicates that $H$ has a large influence on the sensitivity of the sensor. It can be seen from Fig. 4 that the natural frequency and the sensitivity show the opposite trend as $H$ changes. The maximum natural frequency is $16534 \mathrm{~Hz}$, whereas the minimum value is $15346 \mathrm{~Hz}$. This demonstrates that the change in the natural frequency of the sensor with $H$ is unclear. In accordance with the current domestic MEMS processing capacity and performance requirements, $H$ was chosen to be 0.05 or 0.6 , namely, the distance from the hole to the proof mass was 11 or $132 \mu \mathrm{m}$, respectively.

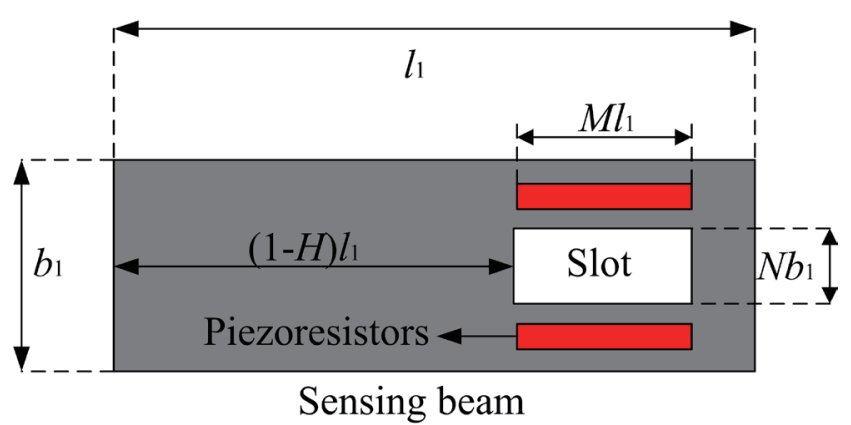

Fig. 2. (Color online) Dimensions of slot and sensitive beam.

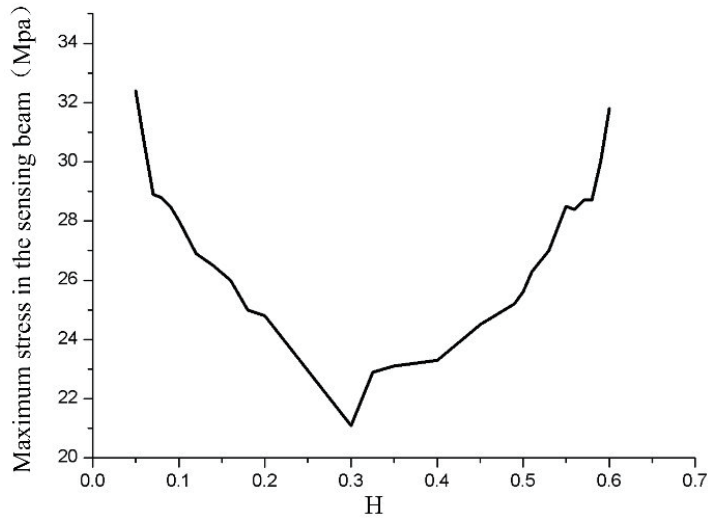

Fig. 3. Effect of $H$ on sensitivity.

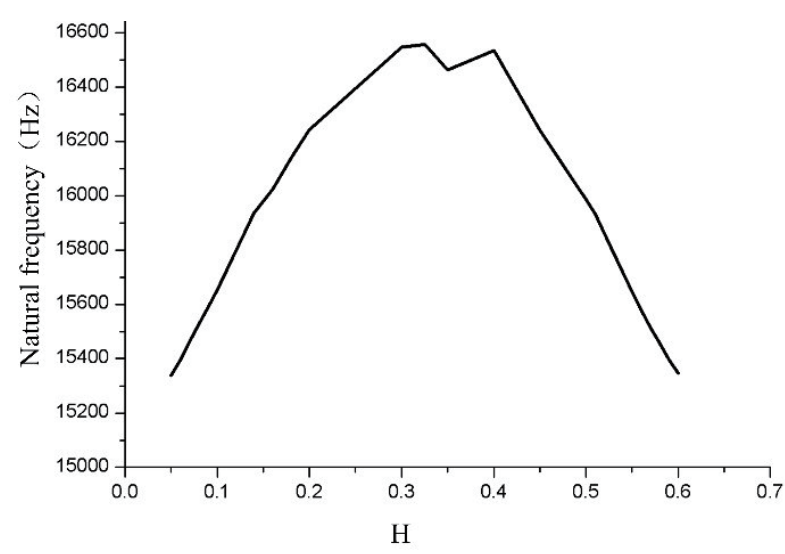

Fig. 4. Effect of $H$ on natural frequency. 


\subsection{Effect of $M$ on sensor performance}

Assuming that $H$ and $N$ are fixed, the static and dynamic characteristics of the sensor are simulated by changing $M$, where $H$ and $N$ are 0.05 and 0.5 , respectively. On the basis of the relationship in Fig. 2 and the MEMS processing capacity, $M$ was varied from 0.1 to 0.9 . Figures 5 and 6 respectively show the influence of $M$ on the sensor sensitivity and natural frequency. As can be seen from Fig. 5, the maximum stress on the beam suddenly changes after the slot is introduced. Then, with increasing hole length, the stress basically remains unchanged. As can be seen from Fig. 6, the natural frequency of the sensor attenuates with increasing length of the slot, but when $M=0.2-0.5$, its natural frequency basically remains stable. Considering the influence of machining precision on sensor performance, $M$ can be selected in the range of 0.2-0.5. Considering the relation between the piezoresistor and the location of the hole, $M$ was chosen as 0.35 , namely, the length of the slot was $77 \mu \mathrm{m}$.

From the above analysis, the detailed parameters of the sensor designed in this study can be obtained. The size of the proof mass is $3000 \times 2500 \times 380 \mu \mathrm{m}^{3}$; the size of the sensing beam is $220 \times 100 \times 22 \mu^{3}$; the size of the suspension beam is $450 \times 150 \times 22 \mu \mathrm{m}^{3}$; the size of the slot is $77 \times 50 \times 22 \mu \mathrm{m}^{3}$. The distance between the slot and the proof mass or the fixed end is $11 \mu \mathrm{m}$. Table 1 shows the simulation results of the natural frequency and maximum stress in the sensing beams. The results showed that the sensitivity of the sensor increased by $54 \%$ and the natural frequency decreased by only $11 \%$ compared with the values for the EB structure, where the latter value is within the acceptable range.

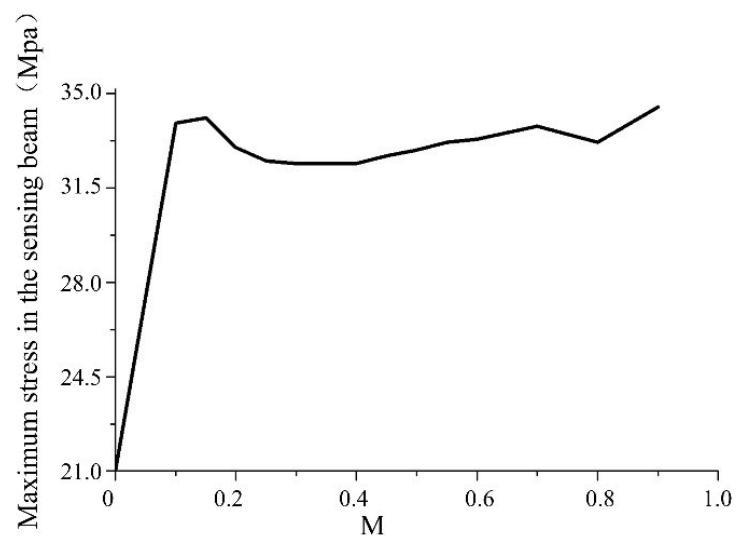

Fig. 5. Effect of $M$ on sensitivity.

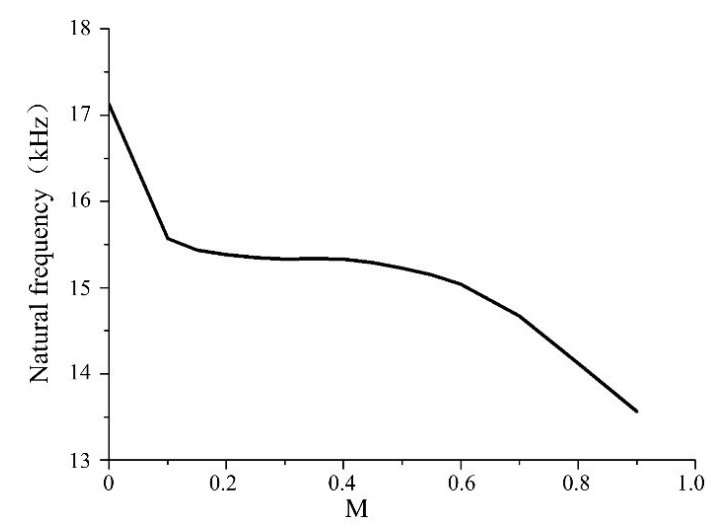

Fig. 6. Effect of $M$ on natural frequency.

Table 1

Performance comparison table of the two structures.

\begin{tabular}{lcc}
\hline Structure & $\begin{array}{c}\text { Maximum stress } \\
(\mathrm{MPa})\end{array}$ & $\begin{array}{c}\text { Natural frequency } \\
(\mathrm{kHz})\end{array}$ \\
\hline EB & 21 & 17.1 \\
SEB & 32.4 & 15.3 \\
\hline
\end{tabular}




\section{Fabrication}

To verify the actual change in the sensor performance after the introduction of slots, sensors with the SEB and EB structures were fabricated on an n-type single-crystal silicon wafer with a resistivity of $2-4 \Omega \cdot \mathrm{cm}$ and a thickness of $380 \mu \mathrm{m}$ for testing and analysis. The fabrication steps are as follows:

(1) The silicon wafers were lightly and heavily doped by ion implantation technology. The heavy impurity doping was first by boron ion diffusion. Then the process of low doping is the same as the process of heavy impurity doping. After these two steps, the piezoresistors were made at the front side of the wafer by photolithography. SEM images of the piezoresistors are shown in Fig. 7(a).

(2) The back-cavity structure of the sensor was processed by RIE technology. From the previously determined parameters, the etching depth was determined to be $358 \mu \mathrm{m}$. The back-cavity structure and side wall morphology after etching are shown in Fig. 7(b).

(3) The glass was corroded to a depth of $20-25 \mu \mathrm{m}$ by a wet process to form a movable space of mass block. Then the glass was bonded with the silicon structure by anode bonding technology.

(4) The lead hole of the sensor chip was formed by a lithography process to ensure a good electrical connection between the piezoresistors and the metal lead. The lead hole was within the resistance bar boundary to ensure effective contact between the metal lead and the bar boundary of the piezoresistors.

(5) Metal leads and electrodes were formed by front sputtering, as shown in Fig. 7(c).

(6) ICP technology was used to etch the top layer of the silicon, releasing the sensitive structure of the sensor so that the proof mass became a movable structure, and the etching clearance width was $12 \mu \mathrm{m}$. The completed sensor chip is shown in Fig. 7(c).

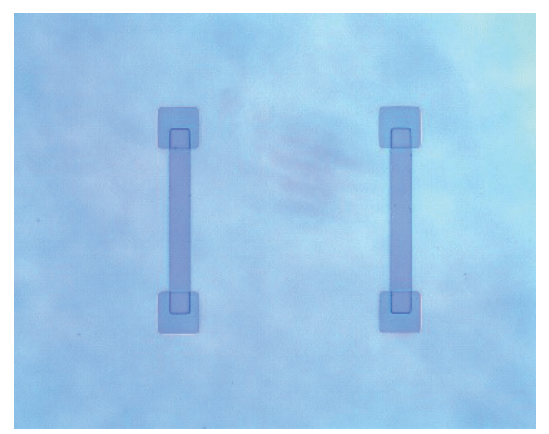

(a)

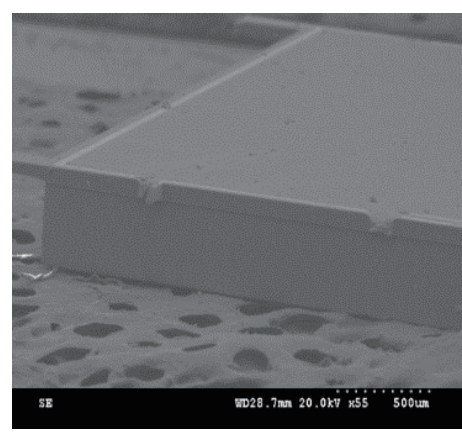

(b)

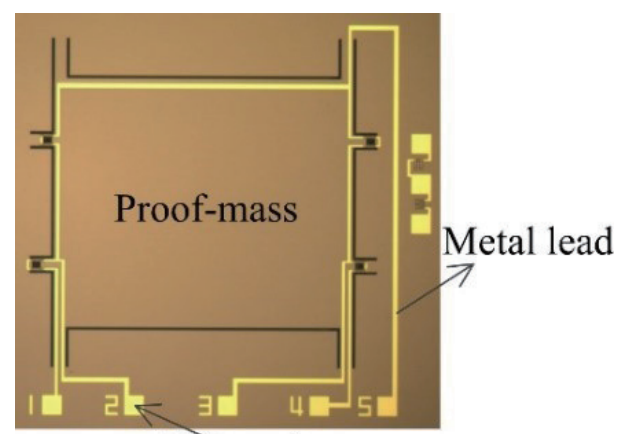

Electrodes

(c)

Fig. 7. (Color online) Key steps of MEMS processing: (a) ion implantation; (b) back-cavity structure of the sensor; (c) finished sensor chip. 


\section{Encapsulation and Testing}

\subsection{Encapsulation}

Because of the small size of the sensor chip and its ease of contamination from the outside environment, the sensor chip must be isolated from the outside environment. The sensor packaging scheme designed in this paper is shown in Fig. 8. The sensor chip was first pasted on a PCB transfer board for electrical connection with the outside world. The stainless-steel shell effectively isolates the sensor chip from the outside environment. Pyrex 7740 glass, which was bonded to the sensor chip, can provide overload protection and damping clearance for the sensor.

\subsection{Testing}

The static calibration system is shown in Fig. 9. The test system mainly includes a centrifuge main engine, working turntable, DC power supply, and multimeter. The available acceleration range of the centrifuge system is 3-200 g, and the acceleration was increased at intervals of $3 \mathrm{~g}$. In order to verify the actual change of the sensor performance after the introduction of slots, sensors with the SEB structure and EB structure were tested. Figure 10 shows the output of the two sensors under different accelerations. The test results show that the sensitivities of the

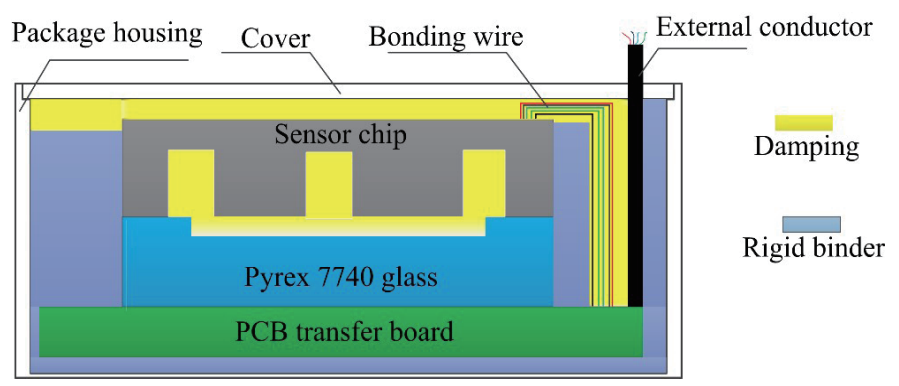

Fig. 8. (Color online) Sensor packaging scheme.

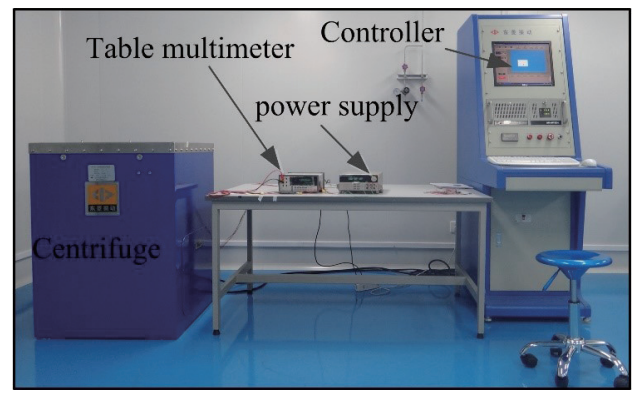

Fig. 9. (Color online) Static calibration system.

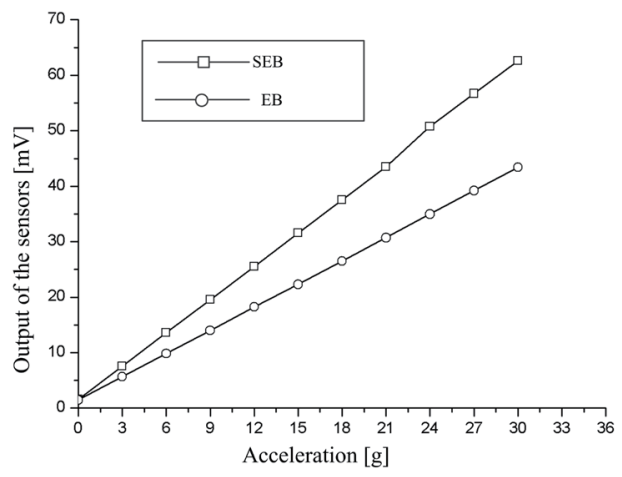

Fig. 10. Output of the two sensors under different accelerations. 
SEB structure and EB structure are 2.03 and $1.4 \mathrm{mV} / \mathrm{g}$ under an $8 \mathrm{~V}$ power supply, respectively. Namely, after introducing the slots, the sensitivity of the sensor increased by $45 \%$.

The dynamic testing system is shown in Fig. 11, which mainly includes a vibrator power amplifier, a signal conditioning circuit, and a computer controller. Sweeping sine signals from the controller actuate the designed sensor and reference sensor, which were fixed on top of the vibrator at the same time. In this paper, the ratio between the outputs of the tested sensor and reference sensor is used to estimate the natural frequency of the designed sensor. When the frequency of the swept signal equals the maximum response frequency of the designed sensor, the output ratio will increase sharply. The results of dynamic testing are shown in Figs. 11 and 12. The test results show that the natural frequencies of the SEB structure and EB structure are 14.76 and $15.16 \mathrm{kHz}$, respectively. This indicates that the natural frequency of the sensor is basically unchanged by the optimization.

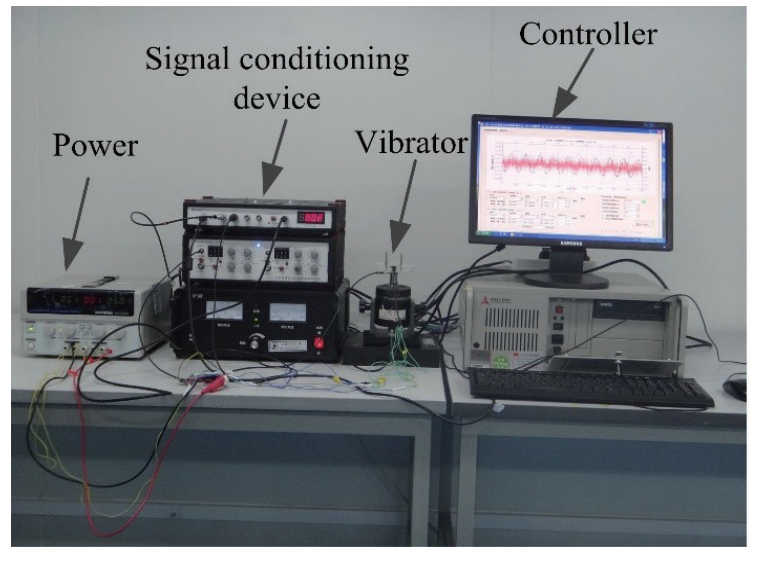

(a)

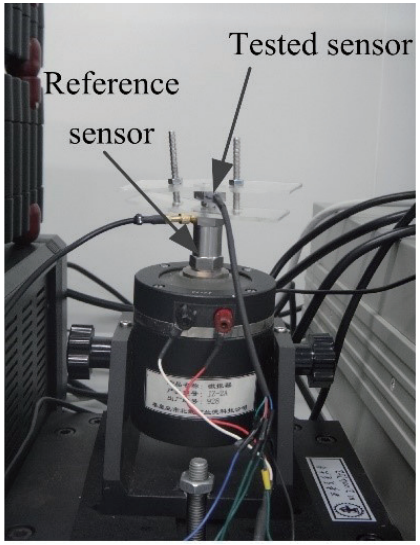

(b)

Fig. 11. (Color online) Dynamic testing system: (a) overall system; (b) vibrator.

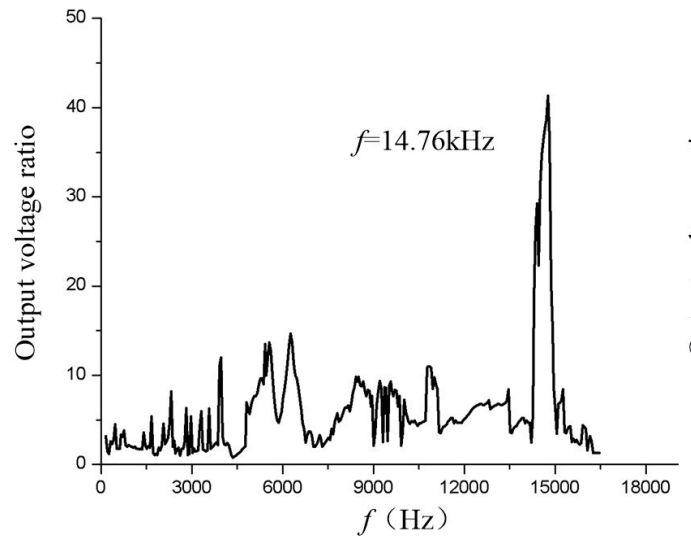

(a)

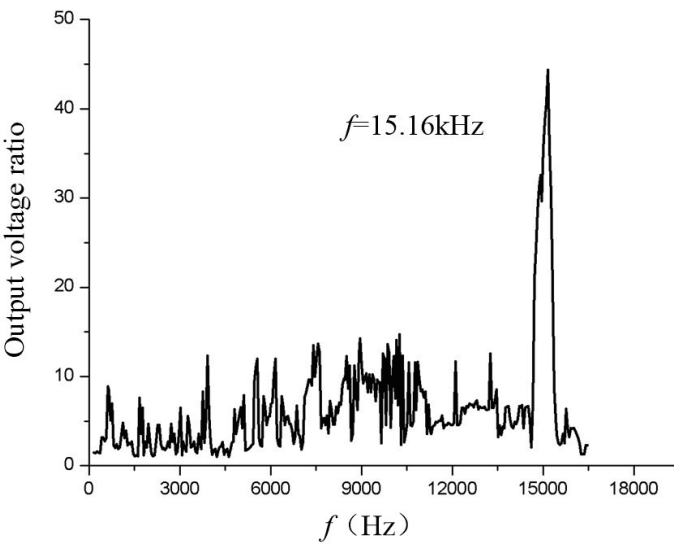

(b)

Fig. 12. Output voltage ratio of the tested sensor and reference sensor: (a) SEB structure; (b) EB structure. 


\section{Conclusions}

(1) A new MEMS silicon piezoresistive accelerometer with a SEB structure was designed and developed to improve the trade-off between the sensitivity and the natural frequency of a piezoresistive accelerometer, which is made possible by incorporating slots into the EB structure.

(2) The mechanism by which the slot location and size influence the performance of the designed structure was studied and analyzed by finite element simulation (FEM).

(3) The developed sensor and a sensor with a conventional unslotted EB structure were fabricated on an n-type single-crystal silicon wafer and packaged for an experiment. The accelerometer prototype was tested in a centrifugal machine and a dynamic calibration system. The experimental results show that the sensitivity of the designed sensor is $2.03 \mathrm{mV} / \mathrm{g}$ and its natural frequency is $14.16 \mathrm{kHz}$. Compared with the structure without slots, the sensitivity was increased by $45 \%$, and the natural frequency remained basically unchanged.

\section{Acknowledgments}

The work presented in this paper was supported by the National Nature Science Foundation of China (Grant Nos. 51605269 and 51275402), Natural Science Basic Research Program of Shaanxi (Grant No. 2020JQ-872), Special Scientific Research Program of Education Department of Shaanxi (Grant No. 20JK0560), and Key Research and Development Program of Shaanxi (Grant No. 2020GY-121).

\section{References}

1 P. D. McFadden and J. D. Smith: Tribol. Int. 17 (1984) 3. https://doi.org/10.1016/0301-679X(84)90076-8.

2 J. M. Dias Pereira, V. Viegas, O. Postolache, and P. S. Girão: Metrol. Meas. Syst. 20 (2013) 465. https://doi. org/10.2478/mms-2013-0040

3 R. X. Gao: Condition Monitoring and Control for Intelligent Manufacturing, R. Yan, S. Sheng, and L. Zhang, eds. (Springer, London, 2006) pp. 167-191.

4 R. Ajith, A. Tewari, D. Gupta, and S. Tallur: IEEE Sens. Lett. 1 (2017) 1. https://doi.org/10.1109/ LSENS.2017.2773652.

5 Z. Baohui, Z. Yan, and S. Yun: China Agric. Inf. 31 (2019) 113. https://doi.org/10.12105/ j.issn.1672-0423.20190412.

6 R. Bogue: Sens. Rev. 33 (2013) 300. https://doi.org/10.1108/SR-05-2013-678

7 B. P. Joshi, A. S. Chaware, and S. A. Gangal: Defence J. 57 (2007) 261. https://doi.org/10.14429/dsj.57.1768.

8 Y. Liu, H. Wang, H. Qin, and Y. Xie: Sens. Rev. 35 (2015) 310. https://doi.org/10.1108/SR-01-2015-0019.

9 Y. Liu, Y. Zhao, W. Wang, L. Sun, and Z. Jiang: Sens. Actuators, A 189 (2013) 8. https://doi.org/10.1016/ j.sna.2012.08.033

10 X. Ma, P. Guo, X. Tong, Y. Zhao, Q. Zhang, P. Ke, and A. Wang: Appl. Phys. Lett. 114 (2019) 253502. https:// doi.org/10.1063/1.5096225.

11 X. Ma, X. Tong, P. Guo, Y. Zhao, Q. Zhang, H. Li, R. Chen, and A. Wang: Sens. Actuators, A 303 (2020) 1. https://doi.org/10.1016/j.sna.2019.111700.

12 Y. Liu, Y. Zhao, B. Tian, L. Sun, Z. Yu, and Z. Jinag: Microsyst. Technol. 20 (2014) 463. https://doi. org/10.1007/s00542-013-1894-9.

13 Y. Wang, D. Mei, and Z. Chen: Sens. Lett. 9 (2011) 1309. https://doi.org/10.1166/sl.2011.1690

14 Y. Zhao, L. Sun, Y. Liu, W. Wang, and B. Tian: Rev. Sci. Instrum. 83 (2012) 784. https://doi. org $/ 10.1063 / 1.4738640$.

15 P. Wang, Y. Zhao, B. Tian, Y. Liu, Z. Wang, C. Li, and Y. Zhao: Meas. Sci. Technol. 28 (2017) 015103. https:// doi.org/10.1088/1361-6501/28/1/015103 


\section{About the Authors}

Peng Wang received his B.S. degree from Xi'an Shiyou University, China, in 2000 and his Ph.D. degree from Xi'an Jiaotong University, China, in 2017. Since 2018, he has been a lecturer at Shaanxi University of Technology, China. His research interests are in MEMS and sensors. (wangpeng1851@163.com)

Nan Wang received his B.S. degree from Shaanxi University of Technology, China, in 2005, his M.S. degree from Xi'an University of Architecture and Technology, China, in 2009, and his Ph.D. degree from Xi'an Jiaotong University, China, in 2013. From 2013 to 2018, he was a lecturer at Shaanxi University of Technology, China. Since 2018, he has been an assistant professor at Shaanxi University of Technology. His research interests are in sensors and the intelligent monitoring and control of mechanical and electrical equipment.

(heroyoyu@126.com)

ChangMing Zhang received his B.S. degree from Shaanxi University of Technology, China, in 2000, his M.S. degree from Taiyuan University of Technology, China, in 2006, and his Ph.D. degree from Xi'an University of Technology, China, in 2020. From 2011 to 2017, he was an associate professor at Shaanxi University of Technology, and since 2017, he has been a professor at Shaanxi University of Technology. His research interests are in aircraft landing gear performance testing and processing technology for difficult-to-machine materials used in aviation. (zhangchangmingsx@126.com)

Fan Yang received his B.S. degree from Sichuan University, China, in 2005 and his M.S. degree from Xidian University, China, in 2010. Since 2005, he has been a lecturer at Shaanxi University of Technology, China. His research interests are in MEMS, bioengineering, and sensors. (yang_fan@snut.edu.cn)

RuiJie Zhao received his B.S. degree from Heze University, China, in 2017. Since 2018, he has been studying for an M.S. degree at Shaanxi University of Technology, China. His research interests are in MEMS and sensors. (2034132180@qq.com) 